\title{
PROPOSTA DE TRATAMENTO DE PROBLEMAS NO GERENCIAMENTO DE PROJETOS
}

\author{
MACÊDO, Gercica \\ Universidade de Pernambuco, e-mail: gcgm@poli.br \\ MELO, Allan \\ Universidade de Pernambuco, e-mail: ajnm@poli.br \\ SILVA, Thaís \\ Universidade de Pernambuco, e-mail: tms3@poli.br \\ MELHADO, Silvio Burratino \\ Universidade de São Paulo, e-mail: silvio.melhado@poli.usp.br \\ SILVA, Simone \\ Universidade de Pernambuco, e-mail: simonerosa@poli.br \\ MONTEIRO, Eliana \\ Universidade de Pernambuco, e-mail: eliana@poli.br
}

\begin{abstract}
RESUMO
As ferramentas da qualidade possuem uma função importante nas análises de problemas relacionados a processos e serviços. Este artigo apresenta um estudo sobre a utilização de ferramentas da qualidade. O objetivo foi identificar fatores que influenciam no gerenciamento de projeto de uma Instituição de Ensino Federal em Pernambuco, através da aplicação de um formulário denominado "Tratamento de Problemas" de forma a criar soluções de melhoria contínua dos processos organizacionais da instituição. A partir da aplicação do formulário foram identificadas três falhas mais frequentes que influenciavam na execução dos serviços contratados pela instituição: Recebimento de projetos com erros técnicos, atraso na entrega de projetos e recebimento de projetos não compatibilizados. Os resultados levaram ao delineamento de alguns planos de ações. Através do formulário, algumas ações foram pontuadas, porém a maior parte dos problemas apresentados necessitaria da colaboração da empresa contratada para identificação mais precisa das causas fundamentais dos problemas. Foi possível observar a dificuldade da equipe técnica da Instituição em interpretar e levantar possíveis causas para os problemas existentes, devido a uma visão conservadora das tecnologias da engenharia e arquitetura, que trazem efeitos negativos como um menor poder interpretativo na análise, deixando despercebido as causas fundamentais dos problemas.
\end{abstract}

Palavras-chave: Qualidade de projeto, tratamento de problemas, ferramenta da qualidade.

\begin{abstract}
Quality tools have an important role in the analysis of issues related to processes and services. This article presents a study about the use of quality tools. The objective was to identify factors that influence in the project management of a Federal institution in Pernambuco by applying a form called "Treatment" in order to create solutions for continuous improvement of the organizational processes of the institution. From the application of the form were identified three more frequent failures that influenced the implementation of the services contracted by the institution: Receipt of projects with technical errors, delays in project delivery and receiving of matched not projects. The results led to the delineation of some action plans. Using the form some actions were punctuated, however most of the problems would require the collaboration of the company hired to carry out more of the fundamental causes of problems. It was possible to observe the difficulty of Institution's technical team in interpreting and find possible causes for the problems,
\end{abstract}

MACÊDO, G.; MELO, A.; SILVA, T.; MELHADO, S.; SILVA, S.; MONTEIRO, E. Proposta de tratamento de problemas no gerenciamento de projetos. In: SIMPÓSIO BRASILEIRO DE QUALIDADE DO PROJETO NO AMBIENTE CONSTRUÍDO, 6., 2019, Uberlândia. Anais... Uberlândia: PPGAU/FAUED/UFU, 2019. p. 510-519. DOI https://doi.org/10.14393/sbqp19048. 
due to a conservative vision of engineering and architecture, bringing negative effects as a minor power interpretive analysis, leaving unnoticed the fundamental causes of the problems.

Keywords: Quality of project, treatment of problems, quality tool.

\section{INTRODUÇÃO}

Segundo Silva (2009) o setor da construção civil se destaca por ser um ambiente de trabalho considerado complexo, apresentando diversidade das atividades, da utilização de máquinas e equipamentos durante o processo de produção, além dos aspectos peculiares que são inerentes ao método produtivo.

O Guia PMBOK (PMI, 2008) define Gerenciamento de Projetos como a aplicação de conhecimento, habilidades, ferramentas e técnicas das atividades de projeto com 0 intuito de atender às expectativas $e$ necessidades das partes envolvidas e interessadas. O Gerenciamento de Projetos também pode ser entendido a partir de seu ciclo de vida, que é composto pelos seguintes processos: iniciação, planejamento, execução, controle e encerramento.

Muita atenção tem sido direcionada ao gerenciamento de projetos, todavia, o histórico de desempenho dos projetos continua insatisfatório com atrasos, estouro no orçamento, má qualidade nos projetos arquitetônicos. De acordo com Caiado \& Salgado (2006), o projeto tem sido avaliado, em diversas pesquisas, como sendo o maior responsável pela falta de qualidade na construção civil. Pesquisas indicam que, em média, $42 \%$ dos defeitos detectados na construção são decorridos de decisões ou ações tomadas ao longo do desenvolvimento do projeto.

A qualidade é o nível de aderência das características do produto e do projeto especificado. O planejamento da qualidade deve focar em garantir que os requisitos aprovados, restrições e legislações sejam atendidas (BRANCO; KEELING, 2014). Zanettini (2002) defende que "não há obra de qualidade sem projeto, ou melhor, sem um bom projeto. Não é possível pensar hoje na cadeia produtiva da construção sem incorporar este aspecto". A qualidade sempre serviu como um diferencial competitivo empresarial. A partir dos trabalhos desenvolvidos dentro das organizações é que os termos relacionados à qualidade vão tomando forma e sendo definidos. Esse sistema foi evoluindo desde a inspeção de produção, para as teorias de gestão e as práticas administrativas. Melhado (1994) foi um dos que organizou as questões de qualidade do projeto e ressaltou a importância da gestão do processo de projeto.

No cenário atual, as organizações buscam outros conceitos existentes como o controle de qualidade, garantia de qualidade e gestão da qualidade, termos que são ligados à qualidade nos processos industriais e serviços, através de indicadores e normas, como a ISO 9001, ISO 14000, PBQPH, entre diversas outras existentes.

De acordo com a NBR ISO 9001 (ABNT, 2008), as alterações de projeto e desenvolvimento devem ser identificadas e registros devem ser mantidos. As alterações devem ser analisadas criticamente, verificadas e validadas, como apropriadas, e aprovadas antes da sua implementação. Devem ser mantidos registros dos resultados da análise crítica de alterações e de quaisquer ações 
necessárias. Dessa forma percebemos que o processo de elaboração, controle e aprovação de projetos estão intrinsecamente ligados ao processo de gestão da qualidade.

O sistema da qualidade possui diversas ferramentas que quando bem aplicadas, garantem a eficácia dos processos organizacionais e consequentemente dos padrões de qualidade como um todo.

O gerenciamento de projetos é utilizado por organizações dos mais diversos ramos de atividade, inclusive na área pública, e tem sido de fundamental importância para transformar o planejamento em resultados, aperfeiçoar a alocação de recursos, diminuir as surpresas, trazendo maior eficiência à gestão de projetos. A utilização de boas práticas em gerenciamento de projetos no setor público é ainda mais recente (PESTANA; VALENTE, 2010).

\section{OBJETIVO}

Este trabalho tem como objetivo identificar os principais fatores que impactam no gerenciamento de projetos das construções de prédios escolares de uma Instituição de Ensino Federal em Pernambuco, e tentar prever a causa raiz dos problemas através de um formulário nomeado de Tratamento de Problemas composto por algumas ferramentas da qualidade.

\section{METODOLOGIA}

A metodologia utilizada neste trabalho subdividiu-se em 4 etapas descritas a seguir: 1) realização de pesquisa bibliográfica relativa ao assunto; 2) Seleção de ferramentas de qualidade para criação do formulário "Tratamento de Problemas"; 3) Levantamento de problemas no gerenciamento de projetos de construção e reforma sob responsabilidade do departamento de obras e projetos da Instituição de Ensino; 4) avaliação dos resultados através da aplicação do formulário criado. Os problemas levantados estão relacionados aos desvios no gerenciamento de projetos. A equipe técnica do departamento é composta por arquitetos e engenheiros.

\section{FERRAMENTAS DA QUALIDADE}

As ferramentas da qualidade são vistas como meios capazes de levar através de seus dados à identificação e compreensão da razão dos problemas e gerar soluções para eliminá-los, buscando a otimização dos processos operacionais da empresa. Pois, para que sejam tomadas ações pertinentes aos problemas ou potenciais problemas, é necessário que seja realizada uma análise dos dados e fatos que precederam ou influenciariam este problema (DANIEL; MURBACK, 2014).

Na NBR ISO (2015), diz que "A organização deve executar ações corretivas para eliminar as causas de não conformidades, de forma a evitar sua repetição". Contanto, os problemas não devem ser apenas interpretados de forma ruim, pois o aprendizado e experiências adquiridas através delas possibilitam a melhoria contínua dos processos que a envolve. 


\subsection{Análise dos fenômenos}

Ao decorrer das atividades, as anormalidades podem vir a ocorrer, porém as suas causas principais nem sempre ficam claras e os processos podem seguir seu fluxo sem que ocorra a correção. Quando se fala em análises de fenômenos, fica implícita a relação direta com o controle de gerenciamento das atividades, com a função de estabelecer procedimentos que assegure a perfeita entrega do que foi planejado.

Os recursos utilizados pelas instituições como orçamentos anual, balanço financeiro, cronogramas, procedimentos gerenciais e operacionais padronizados, e outros dados representados de forma numérica ou gráfica, são importantes fontes para identificação de problemas, além de facilitar a visualização e organização de ideias para identificação da causa Raiz.

\subsection{Brainstorming}

Segundo Leal et al. (2011) brainstorming é uma ferramenta de geração de ideias, que tem sido utilizada com grande frequência nas organizações. Essa ferramenta apresenta uma grande adesão nos setores de qualidade do corpo industrial brasileiro.

O brainstorming é uma conversa com direcionamento específico para solução de um problema, uma troca de sugestões sobre determinado tema. É um método cuja ideia principal consiste na ausência de pré-julgamento. Logo, serão aceitas todas as sugestões.

A fase de condução para a seção de Brainstorming possui três fases conforme Minicucci (2001):

Primeira fase: Exposição de abertura: são dispostos o problema e as informações relevantes que possam ajudar na geração das ideias, deixando claro o objetivo da reunião. Segunda fase: exposição de ideias: é considerada a etapa de produção, onde os participantes efetivamente vão expor suas ideias sobre o problema. Também denominada de fase produtiva. Terceira fase: fase de escrutínio: seleção das ideias emitidas.

Ao concluir a seção e escolha das ideias, um relatório deverá ser gerado, a fim de apontar os problemas e suas respectivas propostas de solução aos superiores responsáveis, para que as propostas sejam analisadas, aprovadas e colocadas em prática, eliminando assim a origem do problema (COLENGUI. 2003).

\subsection{Diagrama de causa e efeito}

O método do diagrama de causa e efeito ou também conhecido como Diagrama de Ishikawa, atua como um guia para a identificação da causa fundamental de um efeito que ocorre em um determinado processo. Este tipo de ferramenta é aplicado em grupos interdisciplinares, de forma que tenha condições de detectar diversas causas para o efeito, sendo que cada participante contribui com seu conhecimento específico (MARTINS et al., 2017).

A premissa básica é a integração dos fatores importantes para o processo analisado. A Figura 1 mostra um exemplo do diagrama de Ishikawa. 
Causas:

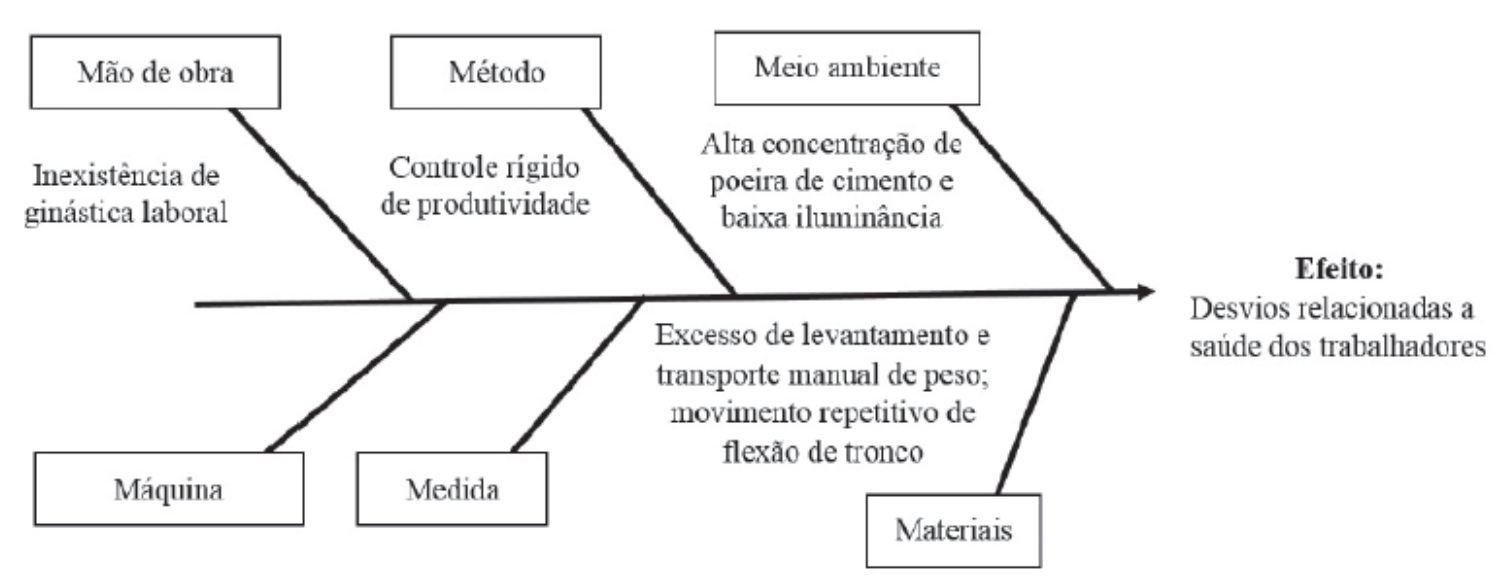

Figura 1- Diagrama de Causa e Efeito

Fonte: Martins et al., 2017

\subsection{Plano de ação}

Para Werkema (1995), essa técnica consiste em descrever o problema, definindo como ele afeta o processo, as pessoas e as consequências posteriores a estas situações. Durante a execução do Plano de Ação, permite saber todos os detalhes de quem é quem, porque está fazendo e o que está fazendo.

Dentro dos diversos modelos de metodologias aplicadas ao plano de ação, o mesmo deve conter alguns dados importantes como:

- Objetivo: Está relacionado com os propósitos das atividades, que podem ser a implantação, alteração ou exclusão de um determinado processo;

- Estratégias: quais os caminhos para o cumprimento do objetivo;

- Cronograma: Apresenta uma relação entre a atividade e o tempo de conclusão da mesma, sendo estimado o tempo necessário de acordo com os recursos disponíveis;

- Responsável: Indica a pessoa ou grupo que irá executar a tarefa;

- Status: É necessário para o acompanhamento das atividades.

\section{IDENTIFICAÇÃO DA INSTITUIÇÃO ANALISADA}

A instituição escolhida para este estudo é vinculada à Rede de Educação Profissional e Tecnológica, criada em 2009, com 17 campi distribuídos do litoral ao sertão de Pernambuco. No organograma, o departamento de obras e projetos é vinculado a Reitoria e é composto por 23 profissionais da área técnica subsidiando todos os campi.

As contratações de serviços de engenharia são regidas pela Lei 8.666 (BRASIL, 1993), de 21 de junho de 1993, que instituiu normas para licitações e contratos da administração pública. O objetivo do departamento de obras e projetos dessa instituição é, na maioria das vezes, fiscalizar a execução dos contratos. Os projetos não são feitos pela equipe técnica, pois as demandas de fiscalização são maiores e o corpo técnico insuficiente para essa atividade. 
O modelo de licitação adotado é o de "menor preço", ou seja, refere-se ao critério de seleção da proposta mais vantajosa para a Administração, que determina o vencedor que será o licitante que apresentar a proposta de acordo com as especificações do edital ou convite, e ofertar o menor preço. Apesar da Instituição não analisar especificamente o critério de "melhor técnica", são colocadas algumas restrições para a contratação. Com relação à arquitetura, a empresa licitada deverá ter realizado no mínimo $16000 \mathrm{~m}^{2}$ de projeto, e as áreas devem ter no mínimo $1000 \mathrm{~m}^{2}$, além disso, a exigência é que os projetos sejam de prédios comerciais, escolas, obras públicas, e que não seja de obra residencial, para que se assemelhe com a realidade. Atendendo a esse requisito, o preço será levado em consideração.

Diferentes de obras privadas, os projetos e orçamentos precisam ser precisos, pois caso tenha falhas, pendências, os profissionais serão cobrados quanto ao Tribunal de Contas da União. A burocracia na obra pública é maior, o projeto precisa retratar a realidade para que seja fidedigno ao que será executado.

Com relação a alteração dos contratos, o artigo 65 da Lei 8.666 (BRASIL, 1993), de 21 de junho de 1993, menciona:

$\S 1^{\circ} \mathrm{O}$ contratado fica obrigado a aceitar, nas mesmas condições contratuais, os acréscimos ou supressões que se fizerem nas obras, serviços ou compras, até $25 \%$ (vinte e cinco por cento) do valor inicial atualizado do contrato, e no caso particular de reforma de edifício ou de equipamento, até o limite de $50 \%$ (cinquenta por cento) para os seus acréscimos.

Sendo assim, as obras em órgãos públicos têm diversas particularidades que devem ser observadas para que a execução seja realizada de acordo com o planejado.

\section{FORMULÁRIO TRATAMENTO DE PROBLEMAS}

O formulário proposto "Tratamento de Problema" é subdividido em cinco etapas, onde a primeira é a identificação do problema ocorrido assim como os processos afetados, os efeitos negativos causados, o local da ocorrência e se houve algum indicador afetado (caso a instituição possua indicadores). As quatro etapas seguintes correspondem às ferramentas de qualidade, análise de fenômenos, Brainstorming, Diagrama de causa e efeito e Plano de ação. A união dessas ferramentas possibilita que o problema seja melhor analisado e discutido, afim de identificar a causa raiz e sua possível solução.

A figura 3 apresenta a estrutura do formulário que foi aplicado a três problemas identificados no departamento de obras e projetos da Instituição de ensino analisada. 


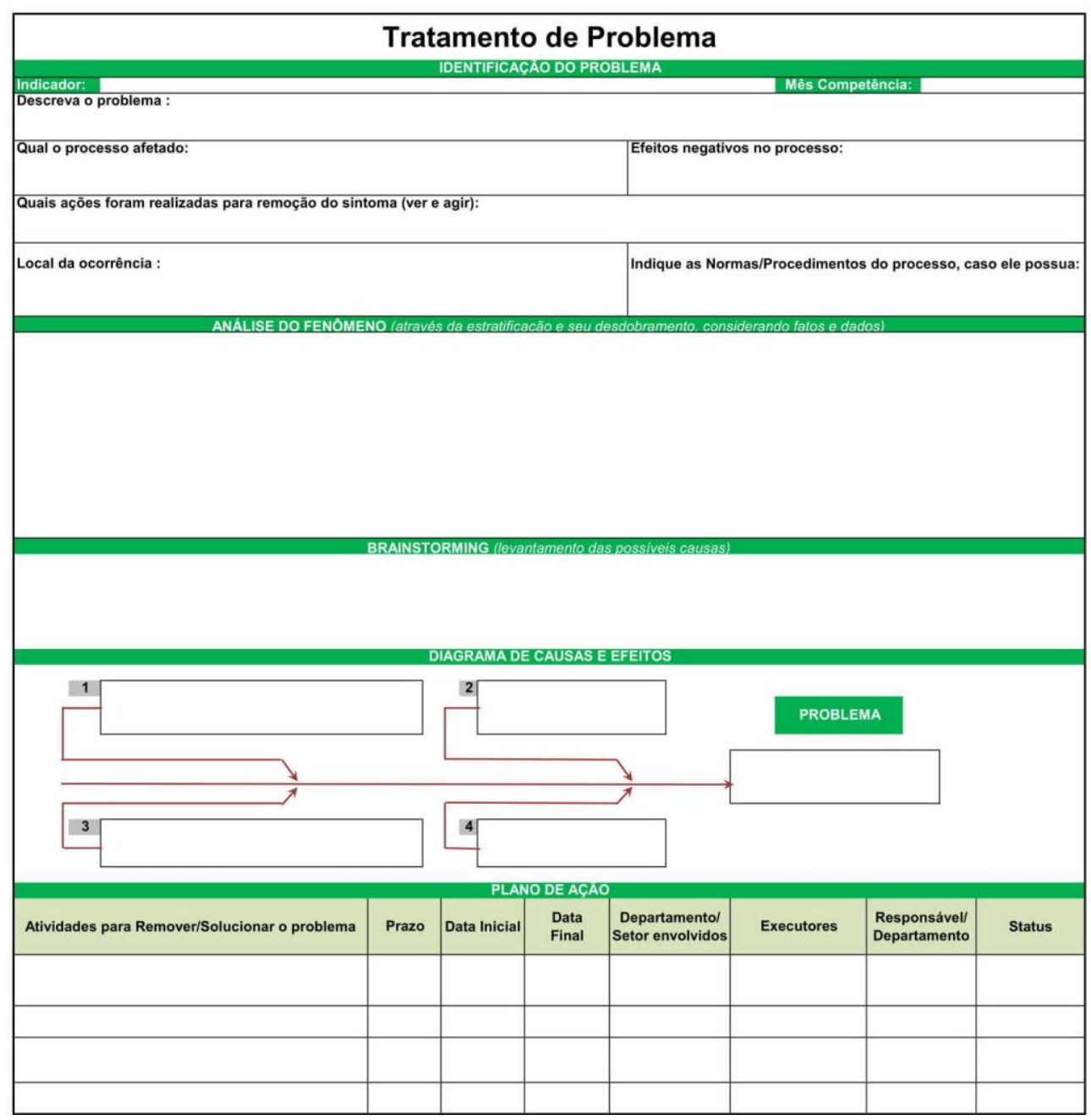

Figura 3 - Formulário "tratamento de Problema"

Fonte: Autores

\section{RESULTADOS E DISCUSSÃO}

O formulário foi aplicado em três problemas identificados pela equipe técnica da Instituição de ensino em estudo. Todos estão associados a prestação de serviço das empresas contratadas por licitação para realização dos projetos de arquitetura e complementares, sob responsabilidade da Instituição contratante, a análise e gerenciamento.

Inicialmente buscou-se identificar os problemas com maior número de ocorrência, conforme orientação da equipe técnica local, dos quais foram apresentados os problemas de:

1. Recebimento de projetos com erros técnicos;

2. Atraso na entrega de projetos;

3. Recebimento de projetos complementares não compatibilizados.

Com o auxílio do formulário "Tratamento de Problemas" foi realizado o detalhamento do problema a fim de se encontrar a causa Raiz. Vale ressaltar que a visão do problema exposto é unilateral, sendo apresentado apenas pela instituição contratante, sem a contribuição da empresa contratada para a realização dos projetos. 
O primeiro problema analisado foi o recebimento de projetos com erros técnicos. Essas falhas afetam o cumprimento dos prazos pré-estabelecidos para entrega final dos projetos. O departamento de projetos da instituição realiza as análises dos projetos recebidos e na ocorrência de divergências, as mesmas são pontuadas através de e-mail à empresa contratada, não possuindo nenhuma outra base para a análise dos fenômenos. O Brainstorming junto à equipe levantou as hipóteses das possíveis causas da presença de falhas nos projetos entregues pela contratada, como: não possuir experiência com a tipologia predial escolar; Mão de obra não especializada; Não conhecimento de escopo do projeto previamente. Através do diagrama de causa e efeito foi evidenciado como causa raiz a falta de um escopo detalhado sobre as necessidades de projeto. Deste modo, o plano de ação proposto para solução do problema teve como sugestões: criar um termo de referência com o escopo de projetos e projeto arquitetônico bem definido antes de submeter à licitação.

O segundo problema levantado foi o atraso na entrega dos projetos, afetando o cronograma previsto no edital de licitação. Como ação imediata (ver e agir) descrita no formulário, na ocorrência desse problema, é realizada as cobranças dos prazos de entrega via e-mail. Não existe nenhum tipo de registro com dados pertinentes aos atrasos para realização da análise dos fenômenos. Dos pontos mais relevantes citados no brainstorming estão a entrega de projetos incompletos e problemas técnicos relacionados ao envio de arquivos computacionais. A aplicação do diagrama de causa e efeito não foi utilizado nessa etapa, pois seria necessária a participação da empresa contratada para melhor compreender os prováveis causadores desta falha e através desta, propor o plano de ação para eliminar o erro. Como proposta de plano de ação para a instituição contratante, é a realização de análise do tempo médio de execução de projetos, a fim de propor maiores prazos em posteriores licitações de projeto.

O último problema analisado foi a entrega de projetos sem a compatibilização entre as disciplinas (arquitetura, elétrica, hidrossanitário, etc.) pela contratada, afetando as datas de entregas previstas no edital de licitação. Falhas nessa etapa também irão propiciar problemas futuros em outras áreas, como a possibilidade de erros na execução das obras por não compatibilidade das disciplinas no projeto. Como no problema anterior, a falta de dados para realizar uma análise de fenômenos que causaram a falha, a unilateralidade das informações sem o auxílio da contratada para apresentar os motivos, torna a análise mais superficial para o Brainstorming e o diagrama de causa e efeito. Uma ação inicial seria agendar acompanhamentos periódicos para aplicação mais precisa do formulário de tratamento de problemas.

Durante o desenvolvimento de cada etapa da aplicação do formulário para identificação da causa raiz dos problemas propostos, foi possível observar a dificuldade da equipe técnica local de interpretar e levantar possíveis causas para os problemas existentes. Por exemplo, na execução do Brainstorming, não houve um número satisfatório de hipóteses para a causa do problema analisado, evidenciando uma visão mais restrita voltada apenas pra soluções mais diretas de engenharia e arquitetura. Essa visão mais conservadora pode ter efeitos negativos, como um menor poder interpretativo na análise, podendo deixar despercebido as verdadeiras caudas do problema. Dessa 
forma, o acompanhamento especializado e treinamento para aprimorar os conhecimentos das ferramentas são primordiais.

Os prazos e responsabilidades para as atividades pertinentes ao plano de ação são delegados aos profissionais da área técnica que possuem contato direto com a atividade fim de onde surgiu o problema. Integrar a empresa contratada para compreender as causas fundamentais dos problemas é uma forma de chegar à raiz do problema de forma mais precisa e assim gerar as melhores ações corretivas. O acompanhamento periódico do cumprimento das ações é fundamental, assim como a continuidade da aplicação desta ferramenta de análise na ocorrência de problemas, para que a eficácia dos processos seja garantida, bem como a melhoria contínua.

\section{CONSIDERAÇÕES FINAIS}

As ferramentas da qualidade possuem uma função importante nas análises de problemas relacionados aos processos e serviços. O gerenciamento de projetos de engenharia e arquitetura, por sua vez, busca atender as necessidades originadas no planejamento do projeto. A criação de ferramentas capazes de dar suporte na gestão através da identificação e tratamento dos problemas relacionados a projetos se torna um diferencial competitivo para uma Instituição.

A utilização de um formulário composto por ferramentas da qualidade aplicadas a gestão de projetos foi aplicada no departamento de obras e projetos em uma Instituição de Ensino Federal em Pernambuco, a fim de identificar os problemas que levavam a diversos erros ao longo dos projetos de obras e reformas dessa Instituição.

Três problemas foram identificados e trabalhados nesse artigo, o recebimento de projetos com erros técnicos, o atraso na entrega de projetos e recebimento de projetos não compatibilizados. Através do formulário, algumas ações foram pontuadas, porém a maior parte dos problemas apresentados necessitaria da colaboração da empresa contratada para uma análise mais precisa das causas fundamentais dos problemas. Foi possível observar a dificuldade da equipe técnica da Instituição em interpretar e levantar possíveis causas para os problemas existentes, que, com uma visão mais conservadora pode ter efeitos negativos como um menor poder interpretativo na análise, podendo deixar despercebido as verdadeiras caudas do problema.

\section{REFERÊNCIAS}

BRASIL. Lei n 8666, de 21 de junho de 1993. Normas para licitações e contratos da Administração Pública. Diário Oficial [da ]República Federativa do Brasil. Poder Legislativo, Brasília, DF, 22 jun. 1993. Seção 1, p. 8269.

\section{CAIADO, V. N.S.; SALGADO, M. S. A gestão de contratos e sua influência naqualidade do processo de projeto: estudo de caso em construtoras do Rio de Janeiro. Gestão e tecnologia de projeto, Vol 1, 2006.}

COLENGHI, V. M. O \& M e Qualidade total: Uma interpretação perfeita, $2^{\circ} e d$. Rio de Janeiro: Qualitymark,2003. 
DANIEL, E. A.; MURNACK, F. G. R. Levantamento bibliográfico do uso das ferramentas da qualidade. Gestão e conhecimento, artigo 08, PUC Minas, 2014. ISSN: 1808-6594.

DINSMORE, P. C.; CAVALIERI, A. Como se tornar um profissional em Gerenciamento de Projetos. Rio de Janeiro, Qualitymark, 2005.

ASSOCIAÇÃO BRASILEIRA DE NORMAS TÉCNICAS (ABNT). NBR ISO 9001: Sistemas de Gestão da Qualidade - Requisitos. Rio de Janeiro: ABNT, 2008.

KEELING, R.; BRANCO, R. H. F. Gestão de projetos - Uma abordagem global. 3ed, Editora Saraiva, 2014.

LEAL, A. A.; MAIA, M. C. S.; MEDEIROS, D. D.; SARREA JR, E. A Qualidade como diferencial competitivo: Uma proposta para a indústria de embalagens plásticas de alimentos do Recife. Anais. XXXI ENCONTRO DE ENGENHARIA DE PRODUÇÃO. Belo Horizonte, 2011.

MARTINS, J. R.; BACELAR, T. C.; BONFIM, W. B.; RODRIGUES, M. V.; XERES, F. C. Análise ergonômica no transporte manual de cargas: um estudo de caso em uma empresa de produção de cimento. GEPROS. Gestão da Produção, Operações e Sistemas, Bauru, Ano 12, nº 1, 2017, p. 269-283. DOI: 10.15675/gepros.v12il.1627

MELHADO, S. B. Qualidade do projeto na construção de edifícios: aplicação ao caso das empresas de incorporação e construção de edifícios. 1994. 294 f. Tese (Doutorado)-Escola Politécnica da Universidade de São Paulo, São Paulo, 1994.

MINICUCCl, A. Relações Humanas: psicologia das relações interpessoais. 6․ed. São Paulo: Atlas, 2001.

PESTANA, C. V. S.; VALENTE, G. V. P. Gerenciamento de projetos na administração pública: da implantação do escritório de projetos à gestão de portfólio na secretaria de estado de gestão e recursos humanos do Espírito Santo. In: III Congresso Consad de Gestão Pública, Painel 21/081 . 2010.

PMI. Um Guia do Conjunto de Conhecimentos em Gerenciamento de Projetos (Guia PMBOK). 4. ed. Pensilvânia: Project Management Institute, 2008.

SILVA, B. M. V. Segurança do trabalho no projeto de arquitetura: diretrizes para o controle dos riscos de acidentes na fase pós-obra. 2009. 123 f. Dissertação (Mestrado em Engenharia Civil) - Universidade de Pernambuco.

WERKEMA, M.C.C. As ferramentas da qualidade no gerenciamento de processos. 2. ed. Belo Horizonte: UFMG; Fundação Christiano Ottoni, 1995. 108 p.

XAVIER, Carlos Magno da Silva e outros. Metodologia de Gerenciamento de Projetos no Terceiro Setor. Rio de Janeiro: Brasport, 2008.

ZANETTINI, S. Arquitetura, razão e sensibilidade. São Paulo: Edusp, 2002, p. 443. 